\title{
New Insights to Characterize Fluid/Carbonate Rock Interfaces Using Transmission Electron Microscopy with Liquid Cell Holder
}

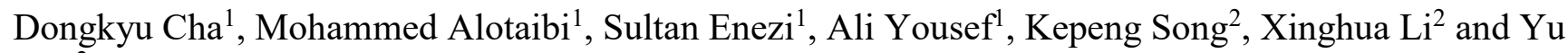 \\ $\operatorname{Han}^{2}$ \\ 1. Saudi Aramco, EXPEC ARC, Dhahran, Saudi Arabia. \\ 2. King Abdulla University of Science and Technology, Physical Science and Engineering Division, \\ Thuwal, Saudi Arabia. \\ * Corresponding author: dongkyu.cha@aramco.com
}

SmartWater flooding showed favorable change in wettability and subsequently increase oil recovery in carbonate reservoirs [1]. Wettability alteration has been proposed as the main reason behind the increase in the recovery due to modified water chemistry, however the exact mechanism is still not fully understood [2]. To address the interaction mechanism, several microscopic to macroscopic conventional methods have been applied such as coreflooding, contact angle, surface charge, NMR and IFT [3]. However, such interaction occurs at a molecular scale where the above mentioned methods cannot provide sufficient information due to limitation of resolution and visualization [4]. Therefore, it is quite challenging to understand the behavior of fluids interactions with carbonate minerals at high resolution.

To overcome these major challenges, Transmission Electron Microscope (TEM) is identified as a novel approach because it can provide both atomic resolution and chemical information at different fluids/rock interfaces. Previously, Cryogenic TEM was mainly used to observe and characterize the liquid sample in ultra-high vacuum. Nevertheless, cryo EM still have an issue that the cryogenic environment used will not mimic the reservoir and consequently obtained results could not be representative.

In this study, liquid cell TEM holder which has encapsulation function features has been applied so that liquid sample can be observed and analyzed close to its native condition. Energy dispersive spectroscopy (EDS) has been utilized to provide the chemical mapping of fluid/fluid and fluid/rock interface. The results provide, for the first time, nano-scale images of oil droplets, calcite nano particles and dissolved salt ions at their native environment. Furthermore, compositional distribution of multiple elements and elemental mapping of the structures in solution are discussed. Figure. 1 demonstrates that it is possible to identify an oil/SmartWater interface in the liquid-cell TEM. EDS analysis shows enrichment of $\mathrm{O}, \mathrm{Cl}, \mathrm{Na}$ and $\mathrm{Mg}$ in the aqueous phase at the fluid/fluid interface. Figure. 2 shows the elemental distribution mapping of fluid/rock interface, in which the elements from SmartWater $(\mathrm{Cl}, \mathrm{Na}$, $\mathrm{Mg}$ ) are mainly distributed on the rock and both $\mathrm{C}$ and $\mathrm{O}$ are observed in the fluid side, suggesting that this is likely an oil/SmartWater/calcite trinary interface.

This nano scale characterization brings a new insight into the chemical distribution of elements and characteristics of interfacial layer at oil/brine interfaces. The presented results demonstrate the potential of liquid-cell TEM in investigating the interactions at fluid/rock interfaces and such understanding would be useful for optimizing the chemistry of injection water to improve oil recovery. 
References:

[1] AA Yousef et al., SPE Reservoir Evaluation \& Engineering 14 (2011), p. 578.

[2] MB Alotaibi, MB and AA Yousef, SPE Reservoir Evaluation \& Engineering 20 (2017), p. 77.

[3] DK Cha et al., SPE Asia Pacific Enhanced Oil Recovery Conference (2015).
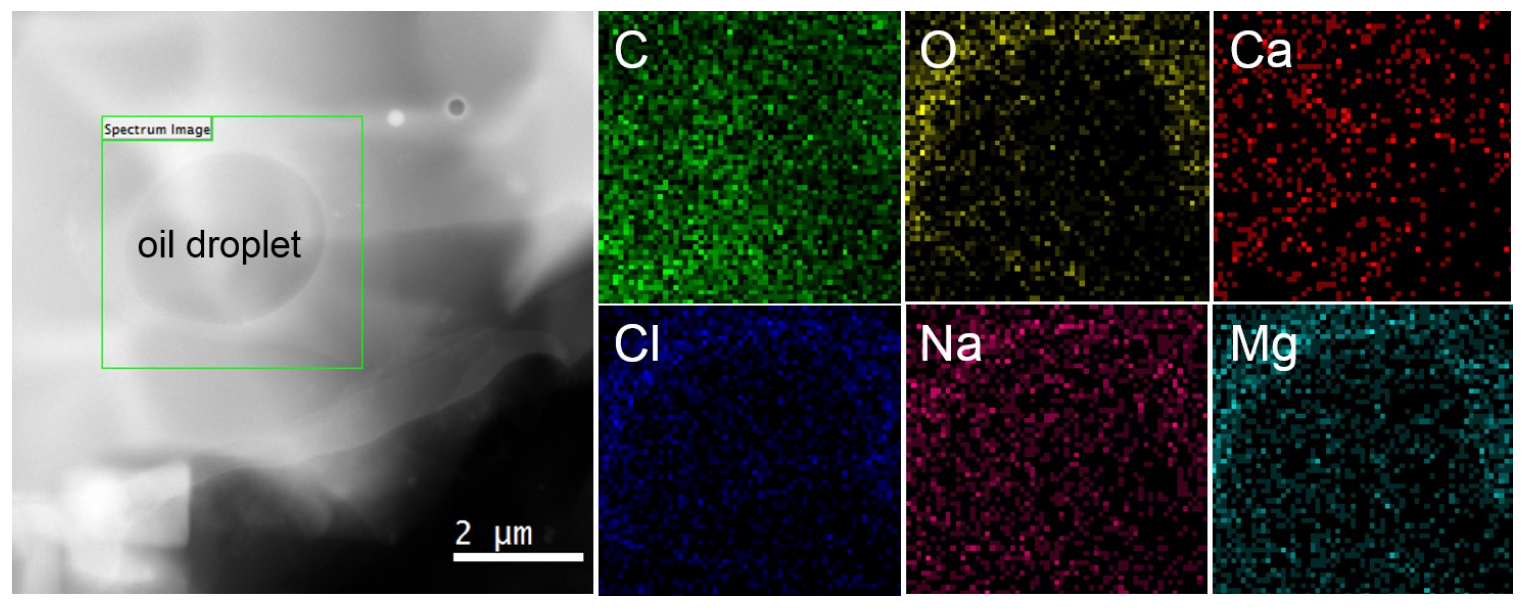

Figure 1. EDS elemental distribution mapping of oil/SmartWater interface under liquid conditions.
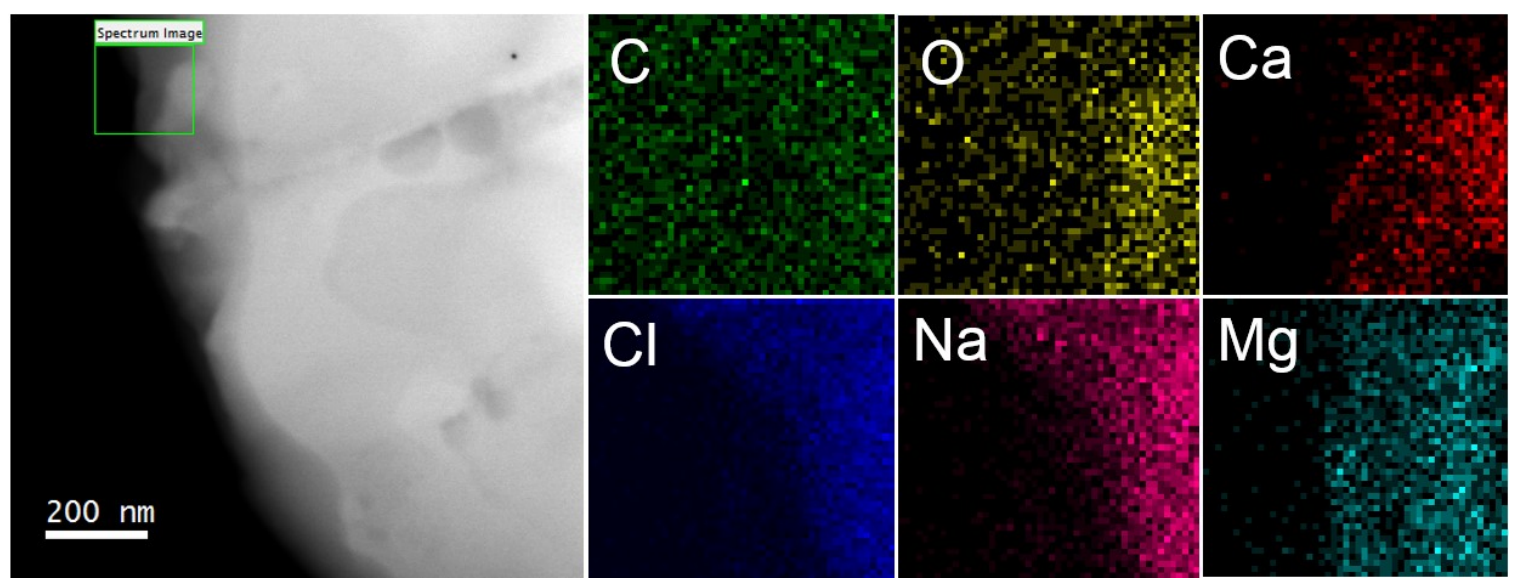

Figure 2. Elemental distribution mapping of fluid/rock interface under liquid conditions. 